\title{
Stability of a composite thick plate with an inhomogeneous field of initial stresses
}

\author{
Vadim V. Eremeev ${ }^{1, *}$, Natalia Y. Shubchinskaya ${ }^{1}$, Denis $V$. Ivashchenko ${ }^{1}$, and \\ Vsevolod A. Minko ${ }^{2}$ \\ ${ }^{1}$ Don State Technical University, Department of Informatics, 34400 Rostov-on-Don, Russia \\ ${ }^{2}$ Belgorod State Technological University named after V.G. Shukhov, 308012 Belgorod, Russia
}

\begin{abstract}
We discuss the linear stability analysis for a rectangular twolayered elastic plate. Each layer of the plate was obtained due to inflation of an annular wedge of a cylinder. As a result, in the plate there are initial stresses. The main aim of the paper is to analyse the influence of the initial stresses on the buckling. Here we use the 3D nonlinear elasticity technique. The linearized boundary-value problem is derived and its non-trivial solutions were obtained.
\end{abstract}

\section{Introduction}

The most of works devoted to the stability of three-dimensional nonlinear elastic bodies concerns homogeneous materials [1-4]. The number of papers dealing with composite bodies, as well as bodies in which the initial stresses do not coincide each other, is much less, see, e.g., [5-7] and the reference therein. The aim of this paper is to study some problems for composite thick plates in which the layers are obtained as a result of inflation of a sector of a cylinder, which generates an inhomogeneous field of initial stresses. Among practical examples of such structures are two-layer slabs, composed of different materials, taking into account technological stresses. For example, it may be a metal film deposited on a flexible polymer substrate. Such problems are important for the development of flexible electronics devices. For such problems, an essential element is the three-dimensional analysis carried out within the framework of the non-linear theory of elasticity. The peculiarity of these problems is the absence of a unique unstressed reference configuration, since for each layer there is different reference configuration, in general. Solutions of these kinds of problems are practically unknown in the literature, which makes this research quite actual.

In this study, we considered the problem of buckling a composite rectangular plate formed by two layers. The resulting plate is symmetrical in thickness. Each layer was obtained as a result of an inflation of a sector of a circular cylinder. For incompressible materials this deformation is universal $[1,8]$, in other words, the form of the solution does not depend on the choice of the material model. Here we chose the incompressible material of Treloar (neoHookean material) as a model of the material [1]. In this paper, the basics of the method of imposing small deformations on finite strains are presented, which provides the stability analysis of equilibrium, see [1-3, 5,9] for details. With conservative external loads, the static

${ }^{*}$ Corresponding author: er.vadim@gmail.com 
stability of an arbitrary prescribed state of a nonlinearly elastic body can be investigated on the basis of the linearization method, also called the static Euler's method. Linearized equilibrium equations and boundary conditions, supplemented by the linearized incompressibility condition are derived. The solutions of the latter were constructed by a semi-analytic method. The critical values of the loading parameters are analysed depending on the geometry of the problem and the properties of the material. The results of the study show that the initial stresses change not only the values of the critical loads, but also the instability modes.

\section{Statement of the problem}

\subsection{Governing equations}

Let us consider two-layered rectangular plate of thickness $2 h$ with sides $a$ and $b$ in a reference placement. Let each layer has the same thickness $h$. We assume that the layers were obtained after inflation of two sections of a circular cylinder, see Fig. 1. After the flattening the layers attached each other in order to have rectangular plate with specific nonhomogeneous field of initial stresses. The problem of iflation of an elastic bar is called Golovin's problem. For compressible material it was solved in [10].
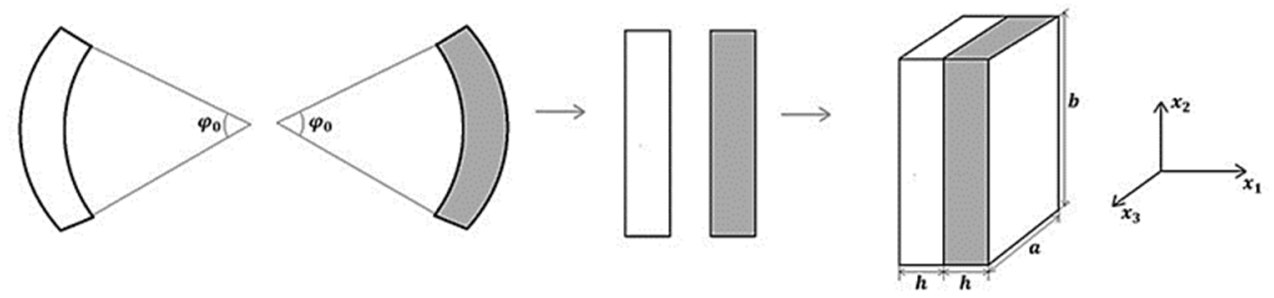

Fig. 1. Rectangular two-layered plate made of two layers after their inflation.

The considered deformation of inflation of an annular wedge is given with the use of Cartesian $X, Y, Z$ and polar coordinates $r, \varphi, z[1,8]$

$$
X=X(r), \quad Y=\chi \varphi, \quad Z=z, \quad \chi>0 .
$$

The governing equations are [1]

$$
\nabla \cdot \mathbf{D}=\mathbf{0}, \quad \mathbf{D}=\mathbf{D}(\mathbf{C})
$$

where $\mathbf{D}$ and $\mathbf{C}$ are the Piola stress tensor and deformation gradient defined as in $[1,11,12]$. Here we also assumed that the mass forces are absent. Let us note that $\mathbf{D}$ and $\mathbf{C}$ depend on the choice of a reference placement. The choice of a reference placement can be done arbitrary but usually they use natural (stress-free) placement. Such choice is not always possible, especially in the case of prestressed compound solids or materials with presstressed inclusions.

With the transformation of an reference placement the deformation gradients transform as follows $[1-3,7]$

$$
\mathbf{C}=\mathbf{P} \cdot \mathbf{C}^{\prime}, \quad \mathbf{C}^{\prime}: \kappa^{\prime} \rightarrow X, \quad \mathbf{C}^{\prime \prime}: \kappa^{\prime \prime} \rightarrow X, \quad \mathbf{P}^{\prime}: \kappa^{\prime} \rightarrow K^{\prime}, \quad \mathbf{P}^{\prime \prime}: \kappa^{\prime \prime} \rightarrow K^{\prime \prime},
$$

where $\kappa^{\prime}$ and $\kappa^{\prime \prime}$ are different initial placements, $K^{\prime}$ and $K^{\prime \prime}$ are intermediate placements, whereas $\mathrm{X}$ is an actual placement. Tensors $\mathbf{P}^{\prime}, \mathbf{P}^{\prime \prime}$ are deformation gradients of the mappings 
from $\kappa^{\prime}$ into $K^{\prime}$ and $\kappa^{\prime \prime}$ into $K^{\prime \prime}$, respectively. Using the incompressibility condition these tensors take the form

$$
\begin{aligned}
& \mathbf{P}^{\prime}=\frac{d X(r)}{d r} \mathbf{e}_{r} \mathbf{i}_{1}+\frac{\chi}{r} \mathbf{e}_{r} \mathbf{i}_{2}+\mathbf{i}_{3} \mathbf{i}_{3}, \\
& \mathbf{P}^{\prime \prime}=-\frac{d X(r)}{d r} \mathbf{e}_{r} \mathbf{i}_{1}+\frac{\chi}{r} \mathbf{e}_{r} \mathbf{i}_{2}+\mathbf{i}_{3} \mathbf{i}_{3},
\end{aligned}
$$

where $\mathbf{e}_{j}$ is the base vectors in polar coordinates in the reference placement, $j=r, \phi, z$, whereas $\mathbf{i}_{k}$ are Cartesian base vectors in the actual placement, $k=1,2,3$.

The compound plate is subjected by normal loading applied to lateral surface $x_{3}=0, a, x_{2}=0, b$ that results in an affine deformation. The faces $x_{1}=-h$ and $x_{1}=h$ are free. As a result, the initial deformed state of the plate is given by the formulae

$$
\begin{aligned}
& \mathbf{C}_{0^{\prime}}=\left(\lambda_{3} \lambda_{2}\right)^{-1} \mathbf{i}_{1} \mathbf{i}_{1}+\lambda_{2} \mathbf{i}_{2} \mathbf{i}_{2}+\lambda_{3} \mathbf{i}_{3} \mathbf{i}_{3}, \quad 0 \leq x_{1} \leq h, \\
& \mathbf{C}_{0^{\prime \prime}}=\left(\lambda_{3} \lambda_{2}\right)^{-1} \lambda_{3} \mathbf{i}_{1} \mathbf{i}_{1}+\lambda_{2} \mathbf{i}_{2} \mathbf{i}_{2}+\lambda_{3} \mathbf{i}_{3} \mathbf{i}_{3},-h \leq x_{1} \leq 0, \\
& \lambda_{3}=\text { const }>0, \lambda_{2}=\text { const }>0 .
\end{aligned}
$$

In (5) $\lambda_{3}, \lambda_{2}$ are stretch parameters.

The linearized boundary conditions take the form

$$
\begin{array}{llll}
x_{3}=0, a: & w_{2}\left(x_{1}, x_{2}, x_{3}\right)=0, & w_{1}\left(x_{1}, x_{2}, x_{3}\right)=0, & \dot{D}_{33}=0, \\
x_{2}=0, b: & w_{1}\left(x_{1}, x_{2}, x_{3}\right)=0, & w_{3}\left(x_{1}, x_{2}, x_{3}\right)=0, & \dot{D}_{22}=0 .
\end{array}
$$

For thin plates conditions (6) corresponds to the simple clamping.

In addition we assume that the layers are attached to each other perfectly that is without slipping, so there is no jump in displacements across the interface between the layers. In this case the linearized boundary conditions are given by

$$
\begin{aligned}
& r=a: u(a, z)=0, \quad \mathbf{e}_{\mathbf{r}} \cdot \dot{\mathbf{D}} \cdot \mathbf{e}_{\mathbf{z}}=0, \\
& x_{3}=-h: \quad \dot{D}_{3 k^{\prime \prime}}=0, \\
& x_{3}=0: \quad \dot{D}_{3 k^{\prime \prime}}=\dot{D}_{3 k^{\prime}}, \quad w_{k^{\prime \prime}}=w_{k^{\prime}}, \\
& x_{3}=h: \quad \dot{D}_{3 k^{\prime}}=0, \quad k=1,2,3 .
\end{aligned}
$$

We use the constitutive relations of the neo-Hookean incompressible material $[1,8]$. The strain energy density and the Piola stress tensor are given by

$$
W=\frac{\mu}{2}\left[\operatorname{tr}\left(\mathbf{C} \cdot \mathbf{C}^{T}\right)-3\right], \quad \mathbf{D}=\mu \mathbf{C}-p \mathbf{C}^{-T},
$$

where $\mu$ is the shear modulus, whereas $p$ is the pressure function defined in an incompressible solids.

Defined with respect to $\kappa^{\prime}$ and $\kappa^{\prime \prime}$ the Piola stress tensors takes the form

$$
\begin{aligned}
& \mathbf{D}^{\prime}=\mu \mathbf{P}^{\prime T} \cdot \mathbf{P}^{\prime} \cdot \mathbf{C}^{\prime}-p^{\prime} \mathbf{C}^{\prime-T}, \\
& \mathbf{D}^{\prime \prime}=\mu \mathbf{P}^{\prime \prime T} \cdot \mathbf{P}^{\prime \prime} \cdot \mathbf{C}^{\prime \prime}-p^{\prime \prime} \mathbf{C}^{\prime \prime-T} .
\end{aligned}
$$


The linearized equilibrium equations are given by [7]

$$
\begin{gathered}
\nabla \cdot \dot{\mathbf{D}}=\mathbf{0}, \\
\dot{\mathbf{D}}^{\prime}=\mu \mathbf{A}^{\prime} \cdot \nabla \mathbf{w}^{\prime}-\dot{p}^{\prime} \mathbf{C}_{0}{ }^{\prime-T}+p_{0}{ }^{\prime} \mathbf{C}_{0}{ }^{\prime-T} \cdot \nabla \mathbf{w}^{\prime} \cdot \mathbf{C}_{0}{ }^{\prime-T}, \quad \mathbf{A}^{\prime}=\mathbf{P}^{\prime T} \cdot \mathbf{P}^{\prime}, \\
\dot{\mathbf{D}}^{\prime \prime}=\mu \mathbf{A}^{\prime \prime} \cdot \nabla \mathbf{w}^{\prime \prime}-\dot{p}^{\prime \prime} \mathbf{C}_{0}{ }^{\prime \prime}+T+p_{0}{ }^{\prime \prime} \mathbf{C}_{0}{ }^{\prime \prime}{ }^{-T} \cdot \nabla \mathbf{w}^{\prime \prime} \cdot \mathbf{C}_{0}{ }^{\prime \prime T}, \quad \mathbf{A}^{\prime \prime}=\mathbf{P}^{\prime \prime T} \cdot \mathbf{P}^{\prime \prime} .
\end{gathered}
$$

In addition for incompressible materials one have to use also the linearized incompressibility condition which is

$$
\operatorname{tr}\left(\mathbf{C}_{0}^{-1} \cdot \nabla \mathbf{w}\right)=0
$$

Thus, the linearized boundary-value problem consists of the linearized equilibrium equations (2), complemented by linearized incompressibility condition (13), boundary conditions (7), expressed with the use of the linearized constitutive equations (11) or (12).

Initial plane stressed state of the plate is given by

$$
\begin{gathered}
D_{11}{ }^{\prime}=\mu \lambda_{3} \lambda_{2}{ }^{-1} \frac{r^{2}}{\chi^{2}}-p_{0}{ }^{\prime} \lambda_{3} \lambda_{2}, \quad D_{22}{ }^{\prime}=\mu \lambda_{2} \frac{\chi^{2}}{r^{2}}-p_{0}{ }^{\prime} \lambda_{2}{ }^{-1}, \\
D_{33}{ }^{\prime}=\mu \lambda_{3}-p_{0}{ }^{\prime} \lambda_{3}{ }^{-1}, \\
D_{11}{ }^{\prime \prime}=\mu \lambda_{3} \lambda_{2}{ }^{-1} \frac{r^{2}}{\chi^{2}}-p_{0}{ }^{\prime \prime} \lambda_{3} \lambda_{2}, \quad D_{22}{ }^{\prime \prime}=\mu \lambda_{2} \frac{\chi^{2}}{r^{2}}-p_{0}{ }^{\prime \prime} \lambda_{2}{ }^{-1}, \\
D_{33}{ }^{\prime \prime}=\mu \lambda_{3}-p_{0}{ }^{\prime \prime} \lambda_{3}{ }^{-1} .
\end{gathered}
$$

The values of $p_{0}^{\prime}$ and $p_{0}^{\prime \prime}$ are found from equilibrium conditions and take the form

$$
p_{0}{ }^{\prime}=\mu \lambda_{3}^{-2} \lambda_{2}^{-2} \frac{r^{2}}{\chi^{2}}, p_{0}{ }^{\prime \prime}=\mu \lambda_{3}^{-2} \lambda_{2}^{-2} \frac{r^{2}}{\chi^{2}} .
$$

From the incompressibility condition (13) we get $\mathrm{X}=\mathrm{X}(r)$ up to additive constant $C$

$$
\mathrm{X}(r)=\frac{r^{2}}{2 \chi}+C .
$$

From (17) we take $r=r(\mathrm{X})$. Finally, parameter $\chi$ is found from the condition

$$
\int_{r_{1}}^{r_{2}} \mathbf{D}_{22}^{\prime} d x_{2}=\int_{r_{1}}^{r_{2}} \mathbf{D}_{22}{ }^{\prime \prime} d x_{2}=0
$$

for $\lambda_{2}=\lambda_{3}=1$. It is given by

$$
\chi=\left[\frac{\mu}{3}\left(r_{2}^{3}-r_{1}^{3}\right)\left(r_{1}^{-1}-r_{2}^{-1}\right)^{-1}\right]^{\frac{1}{4}} .
$$

\subsection{Solution}

The vector of infinitesimal superimposed displacements takes the form

$$
\mathbf{w}=w_{1}\left(x_{1}, x_{2}, x_{3}\right) \mathbf{i}_{1}+w_{2}\left(x_{1}, x_{2}, x_{3}\right) \mathbf{i}_{2}+w_{3}\left(x_{1}, x_{2}, x_{3}\right) \mathbf{i}_{3}
$$


For each layer we are looking for the components of $\mathbf{w}$ and additional pressure with the use of trigonometric functions as follows [7]

$$
\begin{aligned}
& w_{1}=W_{1}\left(x_{1}\right) \sin \frac{\pi n x_{3}}{a} \sin \frac{\pi m x_{2}}{b}, w_{2}=W_{2}\left(x_{1}\right) \sin \frac{\pi n x_{3}}{a} \cos \frac{\pi m x_{2}}{b}, \\
& w_{3}=W_{3}\left(x_{1}\right) \cos \frac{\pi n x_{3}}{a} \sin \frac{\pi m x_{2}}{b}, \dot{p}=P\left(x_{1}\right) \sin \frac{\pi n x_{3}}{a} \sin \frac{\pi m x_{2}}{b} .
\end{aligned}
$$

Eq. (21) is follows from the Fourier method, so it is the exact form of solution which satisfies to the boundary conditions (6) and (7).

Let us represent the linearized Piola stress tensors as follows

$$
\begin{aligned}
& \dot{\mathbf{D}}^{\prime}=\dot{D}_{k s}{ }^{\prime} \mathbf{i}_{k} \mathbf{i}_{s}, \quad k, s=1,2,3 ; \\
& \dot{\mathbf{D}}^{\prime \prime}=\dot{D}_{k s}{ }^{\prime \prime} \mathbf{i}_{k} \mathbf{i}_{s}, \quad k, s=1,2,3 .
\end{aligned}
$$

Since the explicit forms of the tensor components are rather awkward we do not present these expressions here, see [7] for the details.

Substituting (21) into (22) and (23) and then into (10) we obtain the linear system of ordinary differential equations of first and second-order with constant coefficients with respect to unknown functions $W_{1}{ }^{\prime \prime}\left(x_{1}\right), W_{2}{ }^{\prime \prime}\left(x_{1}\right), W_{3}{ }^{\prime \prime}\left(x_{1}\right), P^{\prime \prime}\left(x_{1}\right)$, and $W_{1}{ }^{\prime}\left(x_{1}\right), W_{2}{ }^{\prime}\left(x_{1}\right), W_{3}{ }^{\prime}\left(x_{1}\right)$, $P^{\prime}\left(x_{1}\right)$ defined in corresponding layer. The obtained system is solved analytically, so its general solution was constructed.

In order to consider the plane problem we have $\lambda_{3}=1, n=0$, and $W_{3}=0$. As a result, Eqs. (22) and (23) take more simple form which is not presented here again due to the lack of space.

\section{Results of instability analysis}

Here we consider the loading of the plate in two directions simultaneously. We introduce the stress resultants as follows

$$
N_{k}=\int_{-h}^{h} D_{k k} d x_{3}
$$

$N_{\mathrm{k}}, k=2,3$, are functions of stretch parameters $\lambda_{2}$ and $\lambda_{3}$. Critical values of $\lambda_{2}$ and $\lambda_{3}$ are determined from the condition of existence of non-trivial solutions of the linearized boundary-value problem. So the critical values of the stress resultants are calculated with the use of the of stretch parameters. For any set of natural numbers $m$ and $n$ the critical values of critical values $N_{\mathrm{k}}$ forms a curves in the plane $\left(N_{2}, N_{3}\right)$. These curves split plane $\left(N_{2}, N_{3}\right)$ into the stability area which contains the point $\left(N_{2}, N_{3}\right)=(0,0)$ and instability area, see Fig. 2 . Here we considered a squared plate with dimensionless geometrical parameters $a=b=1, h=0.05$.

\section{Conclusions}

Within the approach of three-dimensional nonlinear elasticity we have discussed the problem of instability of a two-layered plate. The model of the incompressible neo-Hookean material for description of the material properties was used. Both layers of the plate were obtained after inflation of an annular wedge of a circular cylinder. As a result, layers contain nonhomogeneous initial stress field. So for these layers one cannot choose the same natural 
(stress-free) placement. Analysis of the influence of the initial deformation on the critical stress resultants was performed.

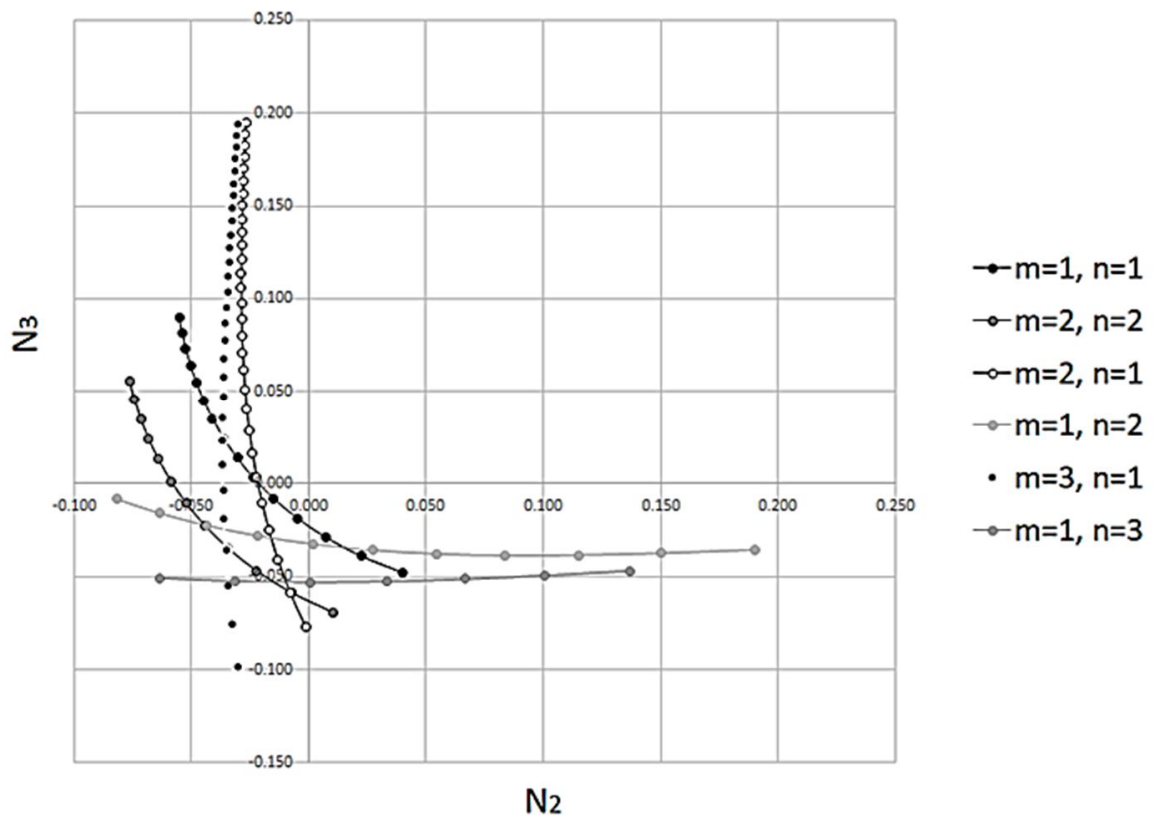

Fig. 2. Critical stress resultants for various buckling modes.

The work was supported by the RFBR within the grant No. 18-31-00135.

\section{References}

1. A.I. Lurie, Nonlinear elasticity (North-Holland, Amsterdam, 1990)

2. A. E. Green, W. Zerna, Theoretical elasticity (Clarendon Press, Oxford, 1954)

3. A. E. Green, J. E. Adkins, Large elastic deformations and non-linear continuum mechanics (Clarendon Press, Oxford, 1960)

4. S. S. Antman, Nonlinear problems of elasticity (Springer, New York, 2005)

5. H. Altenbach, V. A. Eremeyev, Int. J. Non-Lin. Mech., 45, 976 (2010)

6. H. Altenbach, V. A. Eremeyev, Meccanica, 49, 1751 (2014)

7. V. V. Eremeev, L. M. Zubov, Math. Mech. Solids. 22, 773 (2017)

8. C. Truesdell, W. Noll, The nonlinear field theories of mechanics (Springer, Berlin, 2004)

9. Y. B. Fu, R. W. Ogden, Continuum Mech. Thermodyn., 11, 141 (1999)

10. L.M. Zubov, A.G. Popov, Bulletin SSC RAS, 3, 14 (2007)

11. J. G. Simmonds, A brief on tensor analysis (Springer, New Yourk, 1994)

12. L. P. Lebedev, M. J. Cloud, V. A. Eremeyev, Tensor analysis with applications in mechanics (World Scientific, New Jersey, 2010) 